\title{
Calculation of fast pipe diffusion along a dislocation stacking fault ribbon
}

\author{
Xu Zhang and Gang Lu \\ Department of Physics and Astronomy, California State University Northridge, Northridge, California 91330-8268, USA
}

(Received 2 March 2010; revised manuscript received 23 June 2010; published 13 July 2010)

\begin{abstract}
The diffusion of an interstitial Si atom along an edge dislocation in Al is studied by using a quantummechanics/molecular-mechanics coupling method. It is found that the diffusing Si atom follows approximately a sinusoidal trajectory. The diffusion energy barrier along the partial dislocation core is in excellent agreement with the experimental value. We predict that the stacking fault ribbon could provide a fast pathway for diffusion which increases the diffusivity by 6-7 orders of magnitude comparing to the bulk value at $700 \mathrm{~K}$. At the same temperature, the diffusivity along the stacking fault is found to be three to four orders of magnitude greater than that along the partial dislocation core.
\end{abstract}

DOI: 10.1103/PhysRevB.82.012101

PACS number(s): 61.72.Bb, 61.72.Lk

Dislocation core acts as fast paths for diffusing atoms whose mobility can be orders of magnitude higher than in bulk diffusion and this phenomenon is often referred as "pipe diffusion." By creating a short-circuit pathway, pipe diffusion can affect many kinetic processes in bulk materials, including creep, ${ }^{1}$ dynamic-strain aging, ${ }^{2}$ and crystallization. ${ }^{3}$ In thin films and nanocrystals, pipe diffusion can become even more crucial due to the reduced material dimensions. For example, pipe diffusion can significantly influence oxidation, corrosion, recovery of radiation damage, and electromigration in nanoelectronic devices. ${ }^{4}$

Despite its importance, an accurate determination of diffusivity and diffusing mechanism has remained challenging. On the experimental side, owing to the difficulty in tracking atomic motion, very few direct measurements have been reported $^{5,6}$ to date. Among them, a recent experiment on $\mathrm{Si}$ diffusing in $\mathrm{Al}$ stands out. ${ }^{6}$ On the theoretical side, all atomistic simulations have been performed using empirical potentials. For example, the embedded-atom method (EAM) has been widely employed to study pipe diffusion in $\mathrm{Al}^{7} \mathrm{Cu}^{8}$ and Al-Mg alloys. ${ }^{9}$ However, generally speaking, empirical potentials are less accurate in dealing with phenomena involving bond breaking, bond formation, and charge transfer, etc., which are usually present at a dislocation core during pipe diffusion. Moreover, when multiple elements are involved, the empirical potentials such as EAM are even more prone to giving wrong results. On the other hand, although quantum-mechanical simulations are capable of capturing these phenomena, they are often too computationally expensive to deal with dislocations. To the best of our knowledge, there is no literature reporting quantum-mechanical study of pipe diffusion.

Previous studies have shown that the quantum-mechanics (QM)/molecular-mechanics (MM) method can accurately describe the structure of dislocations in $\mathrm{Al} .{ }^{10,11}$ Motivated by the recent experiment which has observed "giant diffusivity" of $\mathrm{Si}$ interstitial pipe diffusion in $\mathrm{Al},{ }^{6}$ we have carried out corresponding QM/MM simulations for $\mathrm{Si}$ diffusions in an edge dislocation in Al. By combining computational efficiency and accuracy, the present QM/MM method links quantum-mechanical simulations based on the Kohn-Sham density-functional theory (KS-DFT) with empirical atomistic modeling based on EAM potential. ${ }^{12,13}$ More specifically, the KS-DFT is applied to the dislocation core where the pipe diffusion takes place; the long-range elastic field of the dislocation on the other hand is handled by the empirical EAM potential. The interaction between the two regions, DFT (region I) and EAM (region II), is treated quantum mechanically with the orbital-free density-function theory (OF-DFT). ${ }^{14,15}$ The total energy of the system is thus expressed as

$$
\begin{aligned}
E_{\text {tot }}\left[\mathbf{R}^{\text {tot }}\right]= & \min _{\rho^{\mathrm{I}}}\left\{E_{\mathrm{KS}}\left[\rho^{\mathrm{I}} ; \mathbf{R}^{\mathrm{I}}\right]+E_{\mathrm{OF}}^{\mathrm{int}}\left[\rho^{\mathrm{I}}, \rho^{\mathrm{II}} ; \mathbf{R}^{\mathrm{I}}, \mathbf{R}^{\mathrm{II}}\right]\right\} \\
& +E_{\mathrm{EAM}}\left[\mathbf{R}^{\mathrm{II}}\right],
\end{aligned}
$$

where $\mathbf{R}^{\text {tot }} \equiv \mathbf{R}^{\mathrm{I}} \cup \mathbf{R}^{\mathrm{II}}$, and $\mathbf{R}^{\mathrm{I}}$ and $\mathbf{R}^{\mathrm{II}}$ denote atomic positions associated with regions I and II, respectively. The three terms on the right-hand side correspond to the energy functional of region I calculated by KS-DFT, the interaction energy between regions I and II which is defined in the following and the energy of region II evaluated by EAM, respectively. The charge density of region II, $\rho^{\mathrm{II}}$ is defined as the superposition of atomic-centered charge densities $\rho^{\text {at }}$ via $\rho^{\mathrm{II}}(\mathbf{r})=\sum_{i \in \mathrm{II}} \rho^{\text {at }}(\mathbf{r}$ $-\mathbf{R}_{i}$ ), which only changes upon the relaxation of region II ions. The charge density of region $\mathrm{I}, \rho^{\mathrm{I}}$, is self-consistently determined for a given ionic configuration by minimizing Eq. (1). The interaction energy formulated by OF-DFT is defined as follows:

$$
\begin{aligned}
E_{\mathrm{OF}}^{\mathrm{int}}\left[\rho^{\mathrm{I}}, \rho^{\mathrm{II}} ; \mathbf{R}^{\mathrm{I}}, \mathbf{R}^{\mathrm{II}}\right]= & E_{\mathrm{OF}}\left[\rho^{\mathrm{tot}} ; \mathbf{R}^{\mathrm{tot}}\right]-E_{\mathrm{OF}}\left[\rho^{\mathrm{I}} ; \mathbf{R}^{\mathrm{I}}\right] \\
& -E_{\mathrm{OF}}\left[\rho^{\mathrm{II}} ; \mathbf{R}^{\mathrm{II}}\right],
\end{aligned}
$$

where the total charge density $\rho^{\text {tot }}$ is given by $\rho^{\text {tot }}=\rho^{\mathrm{I}}+\rho^{\mathrm{II}}$. The three terms on the right-hand side correspond to the energy of the entire system, region I and region II, respectively, all evaluated by OF-DFT. In this work, the planewave pseudopotential method implemented in the VASP package $^{16,17}$ is employed for the KS-DFT simulations of the core region. A plane-wave energy cutoff of $300 \mathrm{eV}$ is used for expanding the wave functions. The projected augmentation wave method ${ }^{18}$ is employed for the pseudopotentials and the Perdew-Zunger local-density approximation ${ }^{19}$ is used to evaluate the exchange-correlation energy. We use WangGovind-Carter kinetic-energy functional with the densitydependent kernel and parameters $\{\alpha, \beta, \gamma\}=\{5 / 6$ $+\sqrt{5} / 6,5 / 6-\sqrt{5} / 6,2.7\}$ (Ref. 15) in the OF-DFT evaluation. The "force-matching" EAM potential of Al (Ref. 20) is em- 


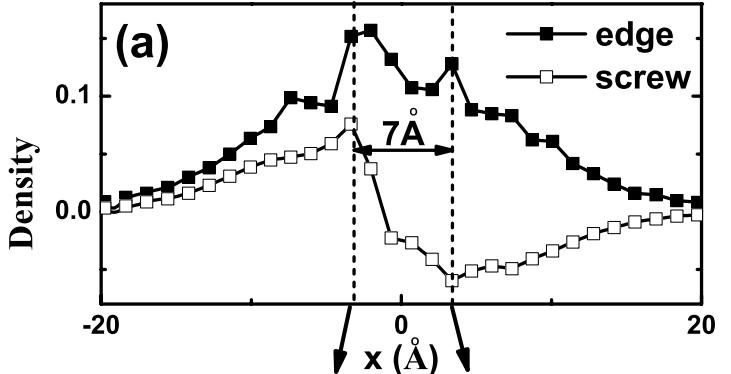

(b) 0000000000000000000

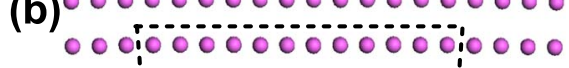

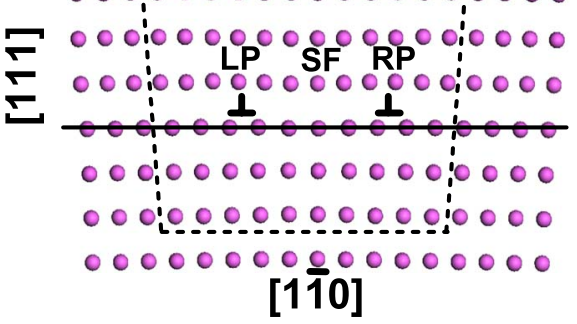

FIG. 1. (Color online) (a) The edge and screw component of the displacement density as a function of $x$. The double peak in the density plot illustrates the dissociation of the perfect edge dislocation into two Shockley partials. The distance between the two partials is $7 \AA$. (b) The atomic structure of the edge dislocation (partially shown) on (11) $)$ plane. LP and RP represent the left and right Shockley partial, respectively, and the stacking fault ribbon is denoted as SF between the partials. The atoms within the dash box are treated by DFT and the others are treated by EAM. The magenta spheres represent $\mathrm{Al}$ atoms.

ployed for the EAM region. The technical details and validations of the QM/MM approach can be found in Refs. 12 and 13. The relaxation for all ions is performed until the maximum force on any ion is less than $0.04 \mathrm{eV} / \AA$.

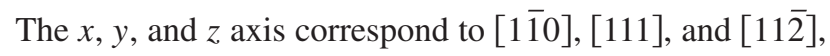
direction, respectively, and the periodic boundary conditions (PBC) are applied in the $z$ direction (along the dislocation line). The entire system consists of $169 \AA \times 69 \AA$ $\times 14.634 \AA$ with 10710 atoms in total. The dimensions of the DFT region are $14.42 \AA \times 11.50 \AA \times 14.634 \AA$ with 198 DFT atoms and the rest of the system belongs to the EAM region, including 10512 EAM atoms. The core of the $\frac{a_{0}}{2}\langle 110\rangle$ edge dislocation is placed at the center of the DFT region, as shown in Fig. 1. The repeating distance along $z$ axis is $\frac{3 \sqrt{6}}{2} a_{0}=14.634 \AA$ ( $a_{0}$ is the lattice constant), which is three times of the minimal period along the $z$ direction $\left(\frac{\sqrt{6}}{2} a_{0}\right)$. Therefore, the fictitious interaction between the periodic images of $\mathrm{Si}$ atoms is negligible - they are separated by $\sim 15 \AA$ A. Since the edge dislocation in $\mathrm{Al}$ dissociates into two equivalent Shockley partials, we consider two "pipes" for the diffusing Si atom: one is along the partial core, and the other is along the stacking fault (SF) ribbon. In addition, we have also examined the "side-way" diffusion of $\mathrm{Si}$ from the left partial core to the stacking fault. The nudged-elastic-band method $^{21}$ has been implemented in the QM/MM approach and is used to determine the diffusion paths and the energy barriers.

First, we calculate the dislocation core structure for the pure $\mathrm{Al}$, which provides hints on plausible locations of the interstitial $\mathrm{Si}$ atoms. Due to the planar structure of the $\mathrm{Al}$ dislocations, we use the relative atomic displacements along $x$ and $z$ directions across the slip plane to represent the edge and screw components of the strain field, respectively. The derivative of the relative displacement with respect to $x$ gives rise to the corresponding dislocation density. ${ }^{22}$ The double peak in the dislocation-density plot suggests that the dislocation is dissociated into two Shockley partials whose position is represented by the peaks. Figure 1 shows the dislocation density and atomic structure of the dislocation core. We find that the partial separation distance is $7 \AA$, which agrees very well to the experimental measurements from the weak-beam transmission-electron microscopy of $8 \AA{ }^{23}$ We note that if the minimal period of $\frac{\sqrt{6}}{2} a_{0}$ is used instead as the thickness of the computational unit cell in $z$ axis, the partial separation distance obtained from the screw component becomes $10 \AA$ whereas the separation distance remains $7 \AA$ from the edge component. This difference suggests when the dislocation dissociates into partials, it may not stay straight; forcing the dislocation straight with the $\mathrm{PBC}$ of the minimal period may introduce small errors in terms of partial separation distance.

Next, we introduce an interstitial $\mathrm{Si}$ atom at the dislocation core, taking cue from the equilibrium dislocation structure. To simulate the pipe diffusion along the dislocation core, we need first to determine the stable energetic positions for the interstitial $\mathrm{Si}$ atom. In this Brief Report, we take the left partial (LP) as an example to study the pipe diffusion as the two partials are equivalent. It is generally believed that interstitial atoms prefer to stay at the tensile region immediately below the slip plane where there is a larger free volume. However, we find it is not true for the studied system. The interstitial $\mathrm{Si}$ atom actually prefers to stay at the same atomic layer of the neighboring $\mathrm{Al}$ atoms, as indicated by the horizontal line in Fig. 1(b). This result is owing to the chemical interaction between $\mathrm{Si}$ and $\mathrm{Al}$ atoms, and is beyond the simple volume considerations. The similar observation is also found in the stacking fault ribbon where the $\mathrm{Si}$ atom is located at the same layer of the $\mathrm{Al}$ atoms. In both cases, the $\mathrm{Si}$ atom has to push aside the neighboring $\mathrm{Al}$ atoms to make space for itself (cf. Figs. 2 and 3). Furthermore, between the stable positions at the partial core (LP) and the SF, the $\mathrm{Si}$ atom is energetically more stable in the former (LP) with an energy difference of $0.08 \mathrm{eV}$. This result may be rationalized by noting that the nearest-neighbor $\mathrm{Al}-\mathrm{Al}$ distance at the $\mathrm{LP}$ is $0.02 \AA$ larger than that at the SF in pure $\mathrm{Al}$.

Figure 2 summarizes the results for the LP. The diffusing $\mathrm{Si}$ atom follows approximately a sinusoidal trajectory that reflects the periodicity of the underlying lattice. The energy profile shows two peaks with the energy barrier of $1.07 \mathrm{eV}$. This energy barrier is in excellent agreement with the experimental value of $1.12 \mathrm{eV}^{6}$ The bonding charge density is defined as the difference between the total solid density and the superposition of atomic densities centered at all atoms. The positive (negative) bonding density indicates charge accumulation (loss) when the atoms are brought together to form a solid. Figure 3 presents the same set of results for SF. The diffusing $\mathrm{Si}$ atom shows the similar periodic pattern of the trajectory as in LP. Interestingly, for both LP and SF, the displacement $\Delta x$ and $\Delta y$ at the saddle point is nearly zero. 


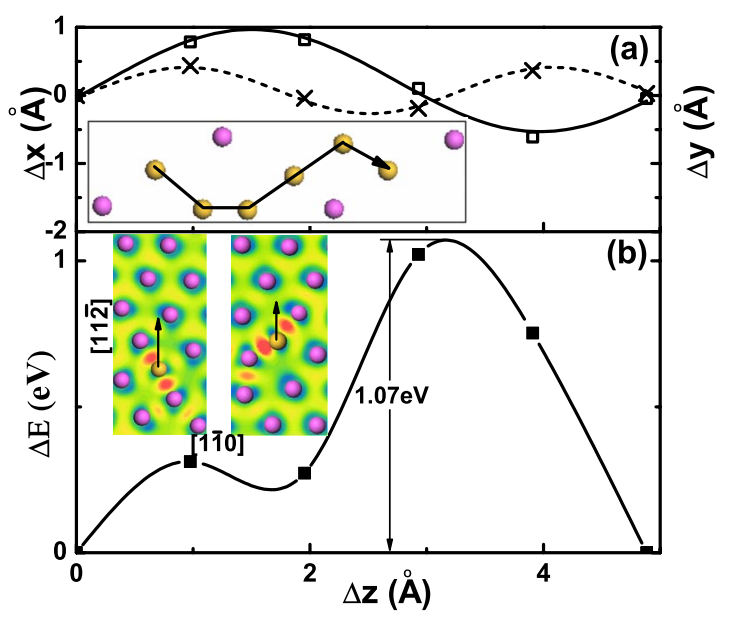

FIG. 2. (Color online) Diffusion of Si along the left partial core. (a) The displacement of the diffusing $\mathrm{Si}$ atom in $x$ direction $(\Delta x$, squares) and $y$ direction ( $\Delta y$, crosses) as a function of the displacement in $z$ direction, $\Delta z$. The displacement is defined relative to the initial $\mathrm{Si}$ position. The solid (dash) line is a sine function that fits the data points of $\Delta x(\Delta y)$. Inset: the schematic trajectory of the $\mathrm{Si}$ atom along the diffusion path on the (111) plane. (b) The energy profile for the diffusion path. The left (right) contour plot represents the bonding charge density around the Si atom at (111) or $x-z$ plane for the initial state (saddle state) and the contour scale ranges from -0.07 (blue) to 0.09 (red). The arrow indicates the diffusion direction. The magenta and yellow spheres represent $\mathrm{Al}$ and $\mathrm{Si}$ atoms, respectively.

The energy profile of SF is symmetric with a much smaller energy barrier of $0.45 \mathrm{eV}$.

To put the results in perspective, we have also calculated the bulk-diffusion energy barrier of $\mathrm{Si}$ in $\mathrm{Al}$. The bulk diffusion of $\mathrm{Si}$ is assisted by an $\mathrm{Al}$ vacancy and the bulk-diffusion energy barrier is the sum of the vacancy formation energy and the energy barrier of Si migration to the nearest-neighbor vacancy. We have thus obtained the bulk-diffusion energy barrier of $1.26 \mathrm{eV}$, comparing to the experimental value of $1.41 \mathrm{eV}^{24}$ Because the bulk-diffusion energy barrier is larger than that along the dislocation core, a significant acceleration of diffusion can be achieved by the pipe diffusion. For example, the Ref. 6 has reported three orders of magnitude increase in diffusivity at $700 \mathrm{~K}$ with the pipe-diffusion energy barrier of $1.12 \mathrm{eV}$-a striking increase in diffusivity dubbed as giant diffusivity. The most surprising and interesting result of the present work is the prediction of a much lower diffusion energy path. We find that by diffusing along the stacking fault ribbon, the increase in diffusivity can be 6-7 orders of magnitude at $700 \mathrm{~K}$-that is three to four orders of magnitude more than the experimental observations of giant diffusivity. The number varies depending on if the theoretical value of $1.26 \mathrm{eV}$ or the experimental value of $1.41 \mathrm{eV}$ is used for the bulk-diffusion energy barrier. In other words, we have predicted a "fast pipe diffusion" which occurs along the stacking fault ribbon. The reason the fast pipe diffusion was not observed experimentally is perhaps because the SF configuration (with $\mathrm{Si}$ ) has a slightly higher energy $(0.08 \mathrm{eV})$ than the LP configuration (with $\mathrm{Si}$ ). This corresponds to a ratio of 1 to 4 for a probability that the $\mathrm{Si}$

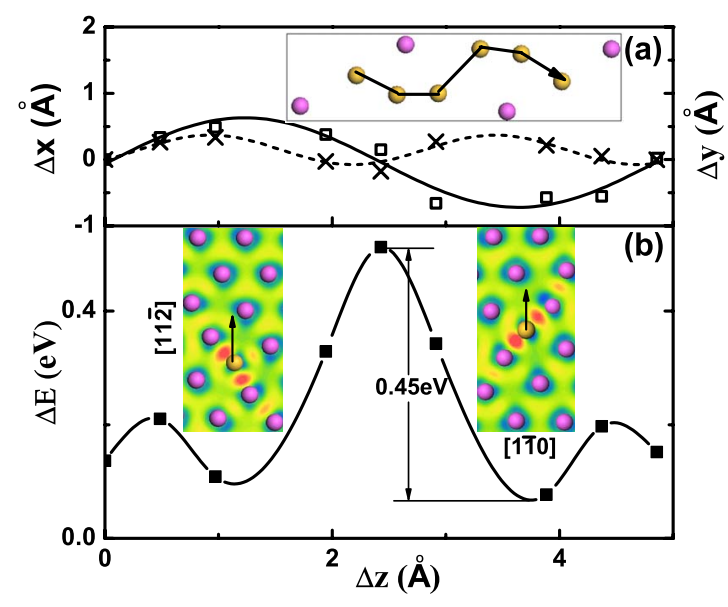

FIG. 3. (Color online) Diffusion of Si along the SF. The caption is the same as in Fig. 2. The energy zero corresponds the energy of the initial state in Fig. 2.

atom ends up at SF vs LP at $700 \mathrm{~K}$ (at room temperature the odds reduce to 1 to 25). However, since the 1 to 4 ratio is not insurmountable, we encourage more experimental measurements to be carried out for this system to verify the prediction. More theoretical work is in progress for other fcc materials.

We can try to understand the results from a structure point of view. As shown in Figs. 2 and 3, the two $\mathrm{Al}$ atoms that are closest to $\mathrm{Si}$ develop a covalent bond with the $\mathrm{Si}$ atom. The average Al-Si bond length is $2.37 \AA$ in the initial state (of the diffusion path) for both LP and SF. However, the equilibrium Al-Si bond length is $2.49 \AA$ According to the experimental measurements of the nearest-neighbor Al-Si distance at $\mathrm{Al} / \mathrm{Si}(111)$ interface. ${ }^{25}$ Therefore the $\mathrm{Al}-\mathrm{Si}$ bonds are compressed during the pipe diffusion and the $\mathrm{Si}$ atom has to push its way through. The schematic trajectory of $\mathrm{Si}$ atom shown in Figs. 2 and 3 also demonstrate that the $\mathrm{Si}$ atom has to

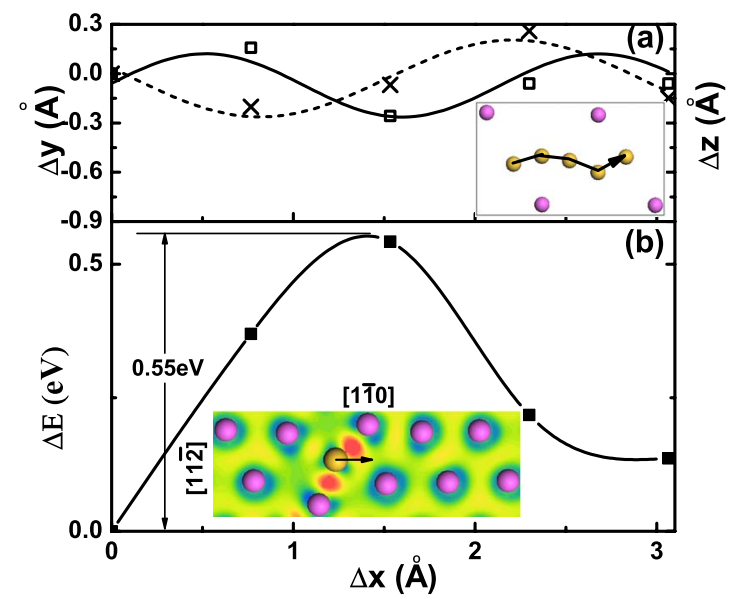

FIG. 4. (Color online) Diffusion of Si from the LP core to the SF ribbon. (a) The solid (dash) line and squares (crosses) denote the displacement of the diffusing Si atom in $y(z)$ direction, $\Delta y(\Delta z)$ as a function of the displacement in $x$ direction, $\Delta x$. (b) The contour plot represents the bonding charge density at the saddle point of the energy profile. See the caption of Fig. 2 for details. 
adjust its trajectory to avoid the neighboring $\mathrm{Al}$ atoms because of the compressed Al-Si bonds. On the other hand, the average Al-Si bond length at the saddle point (of the diffusion path) is $2.33 \AA$ and $2.36 \AA$ for LP and SF, respectively. Therefore the larger average Al-Si bond length in SF at the saddle point makes the diffusion easier than LP.

Finally, we have also examined the diffusion of $\mathrm{Si}$ atom from the LP to the SF ribbon and the results are shown in Fig. 4. It is found that the diffusing $\mathrm{Si}$ atom also follows approximately a sinusoidal trajectory and the energy profile shows one peak with an energy barrier of $0.55 \mathrm{eV}$. The average Al-Si bond length at the saddle point of the diffusion path is $2.37 \AA$. Therefore the $\mathrm{Si}$ atom has to push its way through during the diffusion, which is similar to the pipe diffusions. The side-way diffusion of $\mathrm{Si}$ is relevant to the overall diffusivity because it offers an avenue for $\mathrm{Si}$ atom to take advantage of the fast pipe diffusion along the SF ribbon.
In summary, we have studied the pipe diffusion of a $\mathrm{Si}$ interstitial along the edge dislocation of $\mathrm{Al}$ using a QM/MM method. It is found that the diffusing $\mathrm{Si}$ atom follows approximately a sinusoidal trajectory. The diffusion energy barrier along the partial dislocation core is in excellent agreement with the experimental value. We predict that the stacking fault ribbon could provide a fast pathway for diffusion which increases the diffusivity by 6-7 orders of magnitude relative to the bulk value at $700 \mathrm{~K}$. At the same temperature, the diffusivity along the stacking fault is found to be three to four orders of magnitude greater than that along the partial dislocation core.

This work was supported by NSF under Grant No. DMR0611562 and DOE under Grant No. DE-FC02-06ER25791. The authors acknowledge the NSF Grant No. DMR-0958596 for supporting the computer cluster.
${ }^{1}$ A. B. Pandey, R. S. Mishra, A. G. Paradkar, and Y. R. Mahajan, Acta Mater. 45, 1297 (1997).

${ }^{2}$ Y. Brechet and Y. Estrin, Acta Metall. Mater. 43, 955 (1995).

${ }^{3}$ W. Luo, C. Shen, and Y. Wang, Acta Mater. 55, 2579 (2007).

${ }^{4}$ S. P. Baker, Y. C. Joo, M. P. Knaub, and E. Arzt, Acta Mater. 48, 2199 (2000).

${ }^{5}$ T. E. Volin, K. H. Lie, and R. W. Balluffi, Acta Metall. 19, 263 (1971).

${ }^{6}$ M. Legros, G. Dehm, E. Arzt, and T. J. Balk, Science 319, 1646 (2008).

${ }^{7}$ G. P. Purja Pun and Y. Mishin, Acta Mater. 57, 5531 (2009).

${ }^{8}$ J. Huang, M. Meyer, and V. Pontikis, Phys. Rev. Lett. 63, 628 (1989).

${ }^{9}$ R. C. Picu and D. Zhang, Acta Mater. 52, 161 (2004).

${ }^{10}$ G. Lu, E. B. Tadmor, and E. Kaxiras, Phys. Rev. B 73, 024108 (2006).

${ }^{11}$ Y. Liu, G. Lu, Z. Chen, and N. Kioussis, Modell. Simul. Mater. Sci. Eng. 15, 275 (2007).

${ }^{12}$ X. Zhang and G. Lu, Phys. Rev. B 76, 245111 (2007).

${ }^{13}$ X. Zhang, C. Y. Wang, and G. Lu, Phys. Rev. B 78, 235119 (2008).

${ }^{14}$ Y. A. Wang and E. A. Carter, in Theoretical Methods in Con- densed Phase Chemistry, edited by S. D. Schwartz (Kluwer, Dordrecht, 2000), Chap. 5.

${ }^{15}$ Y. A. Wang, N. Govind, and E. A. Carter, Phys. Rev. B 60, 16350 (1999).

${ }^{16}$ G. Kresse and J. Hafner, Phys. Rev. B 47, 558 (1993); 49, 14251 (1994).

${ }^{17}$ G. Kresse and J. Furthmuller, Phys. Rev. B 54, 11169 (1996).

${ }^{18}$ P. E. Blöchl, Phys. Rev. B 50, 17953 (1994).

${ }^{19}$ J. P. Perdew and A. Zunger, Phys. Rev. B 23, 5048 (1981).

${ }^{20}$ F. Ercolessi and J. Adams, Europhys. Lett. 26, 583 (1994).

${ }^{21}$ G. Henkelman and H. Jónsson, J. Chem. Phys. 113, 9978 (2000).

${ }^{22}$ G. Lu, N. Kioussis, V. V. Bulatov, and E. Kaxiras, Phys. Rev. B 62, 3099 (2000).

${ }^{23}$ W. Höllerbauer and H. P. Karnthaler, Beitrage zur elektronenmikroskopischen Direktabbildung vor Oberflachen 14, 361 (1981).

${ }^{24}$ S. I. Fujikawa, K. I. Hirano, and Y. Fukushima, Metall. Trans. A 9, 1811 (1978).

${ }^{25}$ P. S. Mangat, K. M. Choudhary, D. Kilday, and G. Margaritondo, Phys. Rev. B 44, 6284 (1991). 